\title{
Megaloblastoid Changes Present
}

National Cancer Institute

\section{Source}

National Cancer Institute. Megaloblastoid Changes Present. NCI Thesaurus. Code C82929.

A morphologic finding indicating the presence of abnormally large nucleated erythroblasts in the bone marrow. 